\title{
Effect of Peroxynitrite (ONO0-) on the Function of Murine Perivascular Adipose Tissue
}

\author{
Azizah Binti Ugusman ${ }^{1,2}$, Alex Riddell', Simon Kennedy ${ }^{1 *}$ \\ ${ }^{1}$ Institute of Cardiovascular and Medical Sciences, University of Glasgow, Scotland, UK \\ ${ }^{2}$ Department of Physiology, Faculty of Medicine, National University of Malaysia Medical Centre, Kuala Lumpur, Malaysia \\ Email: *simon.kennedy@glasgow.ac.uk
}

How to cite this paper: Ugusman, A.B., Riddell, A. and Kennedy, S. (2019) Effect of Peroxynitrite $\left(\mathrm{ONOO}^{-}\right)$on the Function of Murine Perivascular Adipose Tissue. Pharmacology \& Pharmacy, 10, 194-204. https://doi.org/10.4236/pp.2019.104017

Received: March 18, 2019

Accepted: April 20, 2019

Published: April 23, 2019

Copyright () 2019 by author(s) and Scientific Research Publishing Inc. This work is licensed under the Creative Commons Attribution International License (CC BY 4.0).

http://creativecommons.org/licenses/by/4.0/

(c) (i) Open Access

\begin{abstract}
Perivascular adipose tissue (PVAT) surrounds the exterior of blood vessels and releases numerous substances such as adiponectin which positively modulate blood vessel tone. In some cardiovascular diseases such as diabetes and high blood pressure, the function of PVAT changes and we speculate that oxidant stress may play a role in this change. PVAT has the ability to generate both superoxide and nitric oxide and these can combine rapidly under physiological conditions to form peroxynitrite $\left(\mathrm{ONOO}^{-}\right)$. In disease states, the production of $\mathrm{ONOO}^{-}$may be increased and so its effect on the function of PVAT is of great interest. Consequently, we studied the effects of acute addition of the oxidant species $\mathrm{ONOO}^{-}$on vascular tone and production of adiponectin by mouse thoracic aortic PVAT. Murine PVAT was immunostained for nitrotyrosine, indicating that $\mathrm{ONOO}^{-}$is formed in the PVAT. Exogenous $\mathrm{ONOO}^{-}$significantly increased the anticontractile effect of PVAT via increased adiponectin content but had no effect on eNOS expression or phosphorylation. These results suggest that generation of $\mathrm{ONOO}^{-}$within PVAT may be an important regulatory mechanism which influences the activity of PVAT. The effect of chronic exposure to raised levels of $\mathrm{ONOO}^{-}$on PVAT function remains to be determined.
\end{abstract}

\section{Keywords}

Perivascular Adipose Tissue (PVAT), Peroxynitrite, Nitric Oxide, Adiponectin, Anticontractile

\section{Introduction}

Perivascular adipose tissue (PVAT) forms the outer layer of all blood vessels with the exception of the cerebral blood vessels and consists of varying proportions of white and brown adipocytes as well as stromal vascular cells. In healthy 
individuals, the PVAT releases a huge variety of paracrine factors, some of which can influence the contractile state of the underlying vascular smooth muscle cells. Since diameter of blood vessels is an important determinant of blood pressure, much research is directed towards understanding how blood vessel diameter is modulated physiologically and how these mechanisms can go awry in diseases such as hypertension. Our recent research has highlighted an important enzyme called AMP-activated protein kinase (AMPK) which is expressed ubiquitously and which may be involved in regulating release of PVAT-derived factors which reduce contractility of the blood vessel. In mice lacking one particular isoform of AMPK, (AMPK $\alpha 1$ knockout mice) the ability of the PVAT to reduce contractility of a large blood vessel, the aorta, was lost [1]. This was due to the PVAT in the knockout mice releasing much lower quantities of an adipocytokine called adiponectin. Adiponectin can induce relaxation of the blood vessel via actions on both the vascular smooth muscle cells in the medial layer of the vessel as well as actions on the endothelial cells which line the blood vessels and are in contact with the blood.

Interestingly, the effect of AMPK $\alpha 1$ knockout can be phenocopied by feeding normal mice a high fat diet for up to 12 weeks [2]. In these mice, the PVAT became inflamed, with infiltration of inflammatory cells, the production of adiponectin was reduced and the ability of the PVAT to reduce contraction was lost. Also, the high-fat diet attenuated phosphorylation of AMPK (a measure of enzyme activity) in the PVAT, linking activity of this enzyme to the beneficial effects of the PVAT [2].

Although our studies are performed in mice, it is interesting to note that obese and insulin resistant patients have inflamed PVAT which secretes inflammatory cytokines. This results in dysfunction of the blood vessel and long-term this may be what increases the risk of high blood pressure and diseases such as atherosclerosis in this group of patients (Reviewed in [3]). In the future, therapy may be targeted at the PVAT or mediators within the PVAT to treat some cardiovascular diseases [4].

Another factor which has been implicated in vascular diseases is oxidant stress. This arises as a result of generation of reactive species as a by-product of biochemical reactions or from the oxidative burst of inflammatory cells. Where there is a focus of inflammation there will be increased levels of oxidant stress and this has been observed around atherosclerotic lesions in mice [5] and humans. Peroxynitrite $\left(\mathrm{ONOO}^{-}\right)$is one such reactive species which can be formed extensively within blood vessels by the reaction of superoxide and nitric oxide; both of which can be generated throughout the wall of the blood vessel and within the PVAT. Peroxynitrite can induce relaxation of blood vessels [6] but can also nitrate proteins and other biological molecules and likely has deleterious effects long-term.

Based on our previous research on the modulating effect of PVAT on vascular function and the strong link between inflammation, oxidant stress and cardio- 
vascular health, in this paper we sought to investigate the following:

1) Does murine PVAT generate nitric oxide (NO) and superoxide and is there evidence that these combine to form $\mathrm{ONOO}^{-}$within the PVAT?

2) Does addition of $\mathrm{ONOO}^{-}$to artery rings with or without attached PVAT affect the ability of the ring to relax?

3) Does addition of $\mathrm{ONOO}^{-}$to PVAT affect the ability of the PVAT to generate beneficial mediators such as nitric oxide and adiponectin and augment relaxation in artery rings?

4) What effect does AMPK $\alpha 1 \mathrm{KO}$ have on generation of $\mathrm{ONOO}^{-}$within the PVAT?

\section{Methods}

\subsection{Generation of NO and Superoxide by PVAT}

Male and female control S129 mice (WT) and mice lacking the $\alpha 1$ isoform of AMPK (KO; weight 20 - $26 \mathrm{~g}$ ) were used for the experiments presented in this paper. A total of 28 WT and 6 KO mice were used in this study. Numbers of animals used in each set of experiments is given in the text and/or figure legends. All animal care and experimental procedures were in accordance with the UK Animals (Scientific Procedures) Act 1986 and data are reported according to the ARRIVE (Animal Research: Reporting of in Vivo Experiments) guidelines. Ethical approval was granted by the University Ethics Committee and conformed to institutional regulations at the University of Glasgow. Mice were bred and housed at University of Glasgow and fed a standard chow diet. All experiments took place during daytime (9am to $6 \mathrm{pm}$ ) in laboratories at Glasgow University. Euthanasia was via exposure to rising $\mathrm{CO}_{2}$ and PVAT was isolated from the mesenteric vessels or the thoracic aorta for fluorescence studies.

Dihydroethidium (DHE) was used to detect superoxide in PVAT. DHE is oxidised by superoxide in the cytosol to form the fluorescent product oxyethidium (excitation $480 \mathrm{~nm}$, emission $567 \mathrm{~nm}$ ). Intact PVAT in Krebs' buffer was incubated in $10^{-5} \mathrm{M} \mathrm{DHE}$ at $37^{\circ} \mathrm{C}$ in a dark, humidified environment for $30 \mathrm{~min}$ utes, washed twice and visualised by confocal fluorescent microscopy (BioRad Radiance 2100). The mean fluorescence intensity of each image was quantified using ImageJ software and values averaged ( $\mathrm{n}>3$ for WT and KO mice). In some experiments, PVATs were co-incubated with superoxide dismutase (SOD; $100 \mathrm{U})$.

NO availability in PVAT was determined using 4,5 diaminofluorescein diacetate (DAF-2DA) using a technique adapted from that used by Gil-Ortega et al. [7]. DAF-2DA is hydrolysed to the impermeable 4,5-diaminofluorescein (DAF-2) by cellular esterases which subsequently reacts with cellular NO to form the fluorescent triazolofluorescein (excitation 495, emission 515). DAF-2DA fluorescence was detected using the $488 \mathrm{~nm}$ argon line for excitation and $530 \pm 30 \mathrm{~nm}$ filter for detection. Samples were visualised using a Bio-Rad 2100 Confocal Laser Scanning microscope with $\mathrm{HeNe}$ laser and $\times 20$ oil/water 
immersion lenses.

\subsection{Nitrotyrosine Detection}

Peroxynitrite reacts with protein tyrosine residues to form stable nitrotyrosine (NT) moieties which can be detected with a specific antibody and used as a marker for $\mathrm{ONOO}^{-}$activity. NT within PVAT was assessed using immunohistochemistry as previously described [5] using a rabbit primary antibody (Millipore), to NT diluted 1:500 in 1\% bovine serum albumin (BSA) in PBS.

\subsection{Small Vessel Wire Myography}

In order to study the effects of PVAT on vascular function and the effect of $\mathrm{ONOO}^{-}$on this, $2 \mathrm{~mm}$ denuded thoracic aortic rings from WT mice either with PVAT left intact or removed were mounted on a wire myograph (Danish Myo Technology) under an optimum tension of $9.8 \mathrm{mN}$ and left to equilibrate for 30 minutes. Vessels were bathed in Krebs' solution at $37^{\circ} \mathrm{C}$ and gassed continuously with $95 \% \mathrm{O}_{2}$ and $5 \% \mathrm{CO}_{2}$. In some experiments either the ring or the isolated PVAT was pretreated with $10^{-4} \mathrm{M} \mathrm{ONOO}^{-}$for 1 hour. Vessels were pre-contracted with $3 \times 10^{-8} \mathrm{M}$ of the thromboxane A2 mimetic U46619. Once tension had stabilised, cumulative dose-response curves to the vasodilator cromakalim were constructed. In some cases, the $\mathrm{ONOO}^{-}$-treated PVAT or the conditioned medium (the solution bathing the PVAT when it was treated with $\mathrm{ONOO}^{-}$) was added into the rings prior to testing relaxation to cromakalim as outlined in our previous studies [1] [2].

\subsection{Adiponectin and eNOS Measurement}

To characterise the effect of $\mathrm{ONOO}^{-}$treatment on eNOS expression in PVAT, aortic PVAT from WT mice $(\mathrm{n}=4)$ was dissected, homogenised and Western blotting was performed. Protein was measured and normalised and samples were subjected to SDS-PAGE using NuPAGE ${ }^{\circledR}$ system. Membranes were probed with with phospho-eNOS (Ser ${ }^{177}$ ) and total eNOS primary antibodies. Detection was via enhanced chemiluminescence. Adiponectin was measured in homogenised PVAT samples $(\mathrm{n}=11)$ using a commercially available ELISA kit as previously described [1].

\subsection{Statistical Analysis}

All data are expressed as the mean \pm SEM, where $n$ equals the number of mice used per experiment. Data were analyzed with GraphPad Prism 5.0 software. For fluorescence data, an unpaired Student's t-test was used to compare mean fluorescence intensity. In other experiments, when comparing two or more variables, two-way ANOVA (analysis of variance) tests were used. When comparing two or more data groups (contraction data), two-way ANOVA followed by Newman-Keuls post hoc test was used. In all cases, a p-value of $<0.05$ was considered statistically significant. For fluorescence data, an unpaired Student's t-test was used to compare mean fluorescence intensity. 


\section{Results and Discussion}

\subsection{NO and Superoxide Formation by PVAT}

To demonstrate that PVAT has the capacity to generate the two species required for formation of $\mathrm{ONOO}^{-}$, we attempted to detect $\mathrm{NO}$ and superoxide within samples of mesenteric PVAT. Figure 1 (upper panel) shows fluorescence due to superoxide formation and the lower panel shows fluorescence attributable to formation of NO within the PVAT. Mice lacking the $\alpha 1$ isoform of AMPK did not appear to generate any greater quantities of either superoxide or NO within the PVAT. Others studies have also found PVAT samples to generate NO [8] and superoxide [9] and that high-fat diet increases superoxide and therefore PVAT oxidant stress. Interestingly, incubation of PVAT from obese animals with the endogenous scavenger enzymes superoxide dismutase (SOD) and catalase restored PVAT function in an obese animal model [10].

In WT mice, pretreating the PVAT with $10^{-4} \mathrm{M} \mathrm{ONOO}^{-}$for one hour significantly increased the detection of superoxide and this effect was inhibited by co-incubating with the dismutating enzyme SOD which detoxifies superoxide in vivo (Figure 2).

We then attempted to assess whether we could detect nitrotyrosine (NT) formation within the PVAT, using this as a footprint for $\mathrm{ONOO}^{-}$formation. We anticipated that we would detect $\mathrm{NT}$ as both $\mathrm{NO}$ and superoxide were detected. Indeed, NT was abundantly expressed and there appeared to be more expression in the KO PVAT than the WT although this was not quantified (Figure 3(a)). Preincubating the PVAT with $10^{-4} \mathrm{M} \mathrm{ONOO}^{-}$for 1 hour markedly increased detection of nitrotyrosine as expected (Figure 3(b)). Other studies such as that by Marchesi et al. [11] have detected peroxynitrite production by mesenteric vessels which was increased in obese mice; however, we believe this study to be the first to report generation of peroxynitrite specifically within the PVAT.

\subsection{Small Vessel Wire Myography}

Next, the effect of $\mathrm{ONOO}^{-}$on the ability of the PVAT to modulate vessel relaxation was studied using small vessel wire myography. Relaxation responses to cromakalim were significantly enhanced in the presence of PVAT (maximal relaxation was $43.73 \% \pm 7.36 \%$ vs. $21.12 \% \pm 0.92 \%$ in PVAT(-) vessels, $\mathrm{p}$ $<0.01)$. This is in line with our previous studies using mouse aortic rings [1] [2]. Pre-treatment of $\mathrm{PVAT}(+)$ aortic rings with $\mathrm{ONOO}^{-}$did not have any effect on the relaxation to cromakalim (Figure 4(a)), however, transferring $\mathrm{ONOO}^{-}$-treated PVAT to PVAT(-) rings enhanced the relaxation to cromakalim (maximal relaxation increased to $64.45 \% \pm 9.21 \%, \mathrm{p}<0.0001$; Figure $4(\mathrm{~b})$ ). Incubation of PVAT(-) rings with conditioned media from $\mathrm{ONOO}^{-}$-treated PVAT also induced significantly more relaxation to cromakalim (maximal relaxation raised to $68.15 \% \pm 9.22 \%, p<0.0001)$. The augmented relaxation observed following addition of $\mathrm{ONOO}^{-}$-conditioned medium or $\mathrm{ONOO}^{-}$-treated PVAT to 
PVAT(-) rings was greater than that caused by the presence of PVAT alone $(\mathrm{p}<$ $0.001)$.

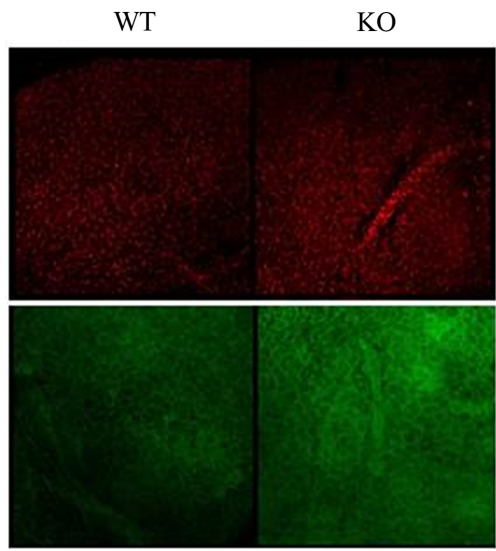

Figure 1. Detection of superoxide (upper panels) and NO (lower panels) using fluorescence in mesenteric PVAT from wild-type (WT) and AMPK $\alpha 1$ knockout (KO) mice. Superoxide was detected using dihydroethidium and NO was detected using DAF-2DA. Images are representative of no less than three independent experiments. No significant difference was seen $(n=3)$.
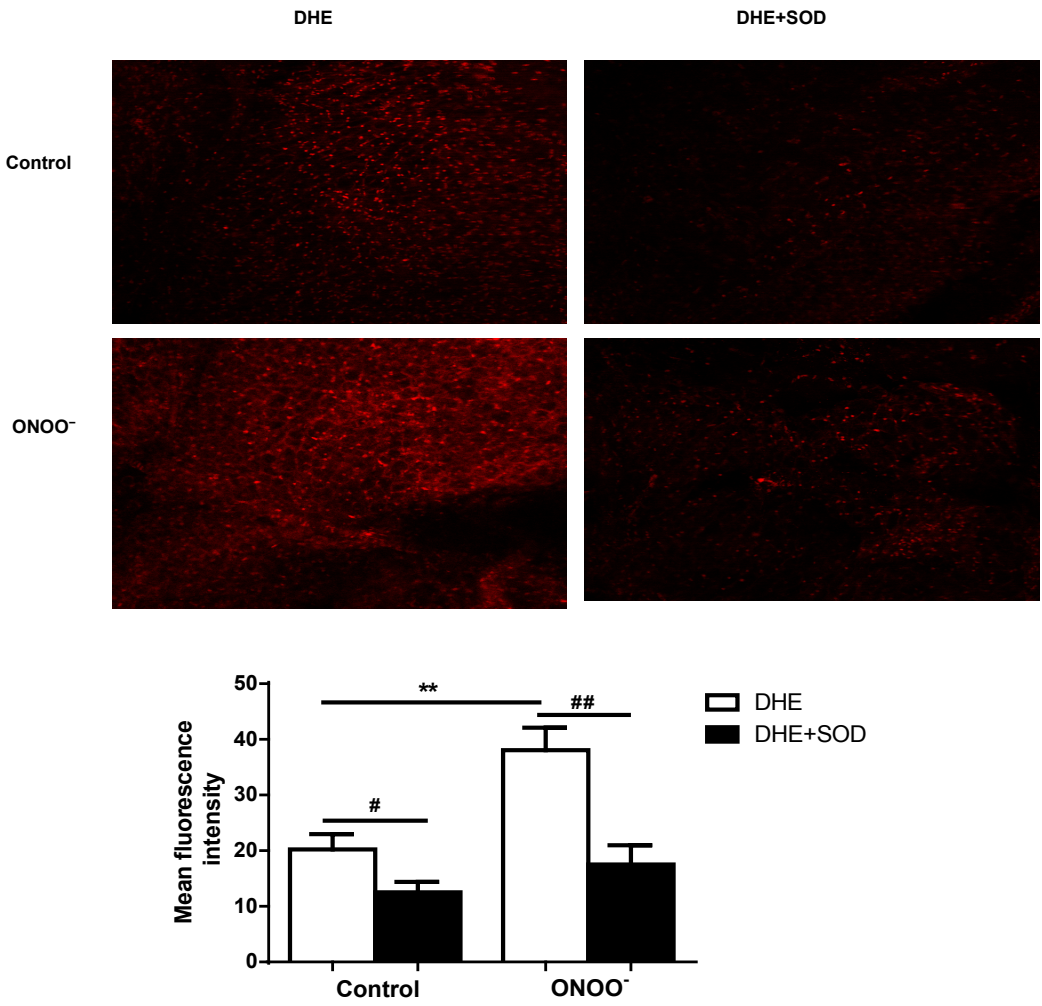

Figure 2. Upper panel-in WT mice, treating PVAT with peroxynitrite $\left(10^{-4} \mathrm{M}\right.$ for 60 mins) increased detection of superoxide compared to control (left side figures) and this was blocked by co-incubating with the dismutating enzyme SOD $(n=5$; right side figures). Lower panel-histogram quantifying the significant increase in measured fluorescence caused by ONOO- and the effect of SOD $(n=5 ; \# p<0.05$ and \#\#p $<0.01$ vs. DHE alone; ${ }^{* *} \mathrm{p}<0.01$ vs. control without $\mathrm{ONOO}^{-}$addition). 


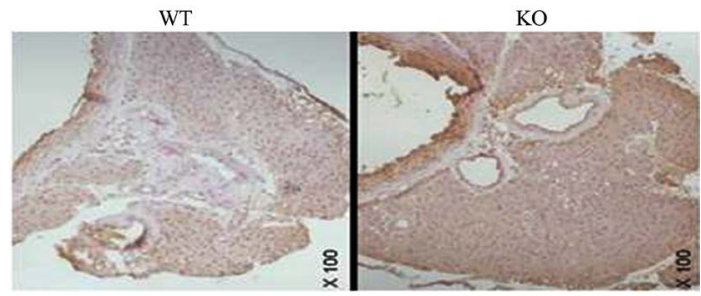

(a)

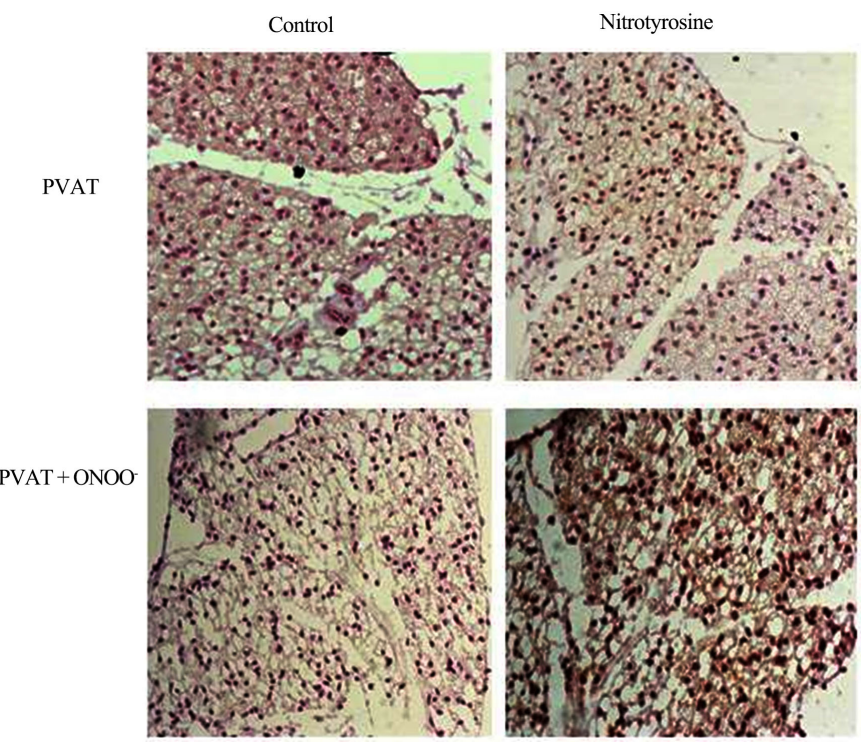

(b)

Figure 3. (a) representative photomicrographs showing nitrotyrosine expression throughout the vessel wall and PVAT in WT mice (left) and AMPK $\alpha 1 \mathrm{KO}$ mice (right). (b) Incubation of the PVAT with $10^{-4} \mathrm{M} \mathrm{ONOO}^{-}$for 1 hour markedly increased detection of nitrotyrosine (Magnification $\times 100$ top panel and $\mathrm{x} 200$ bottom panel).
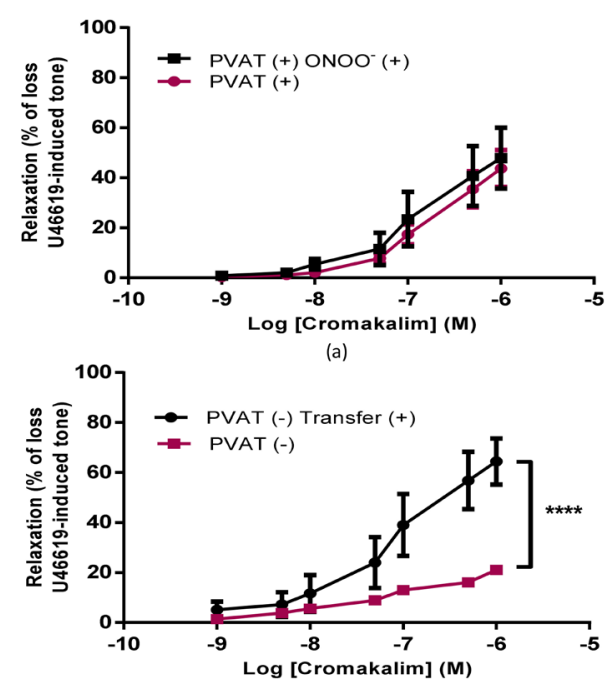

(b)

Figure 4. (a) Treatment of WT mouse aortic rings with intact PVAT with $\mathrm{ONOO}^{-}$did not enhance relaxation to cromakalim. (b) In aortic rings without PVAT, addition of PVAT which had been exposed to $\mathrm{ONOO}^{-}$for 1 hour significantly enhanced relaxation $\left(\mathrm{n}=6 ;{ }^{* * *} \mathrm{p}<0.0001\right.$ vs. PVAT(-)). 
Our results complement those published by Costa et al. [12] who showed that the brown adipose tissue in thoracic aortic PVAT is a potent source of reactive oxygen species (ROS) due to the abundance of mitochondria in the brown adipocytes and that the dismutation product of superoxide-hydrogen peroxide can attenuate contraction. Our data indicates that removal of superoxide by its reaction with NO may also increase the anticontractile effect of PVAT. Since in these studies we used aortic rings with the endothelium removed, the source of the NO is likely to have been the PVAT rather than the vascular endothelium.

\subsection{Adiponectin and eNOS Measurement}

Our previous studies had identified adiponectin, a very abundant adipokine, as being important in mediating the anticontractile effect of murine aortic PVAT. We then speculated that the positive effects of peroxynitrite on the anticontractile effect of aortic PVAT may be mediated via increased generation of adiponectin within the PVAT or perhaps via activation of eNOS, the enzyme which generates the vasodilator $\mathrm{NO}$ and which is present in PVAT [13]. Using samples of PVAT from WT mice, we found that treatment with $\mathrm{ONOO}^{-}$significantly increased adiponectin content as measured by ELISA (Figure 5(a)) while having no significant effect on the expression or phosphorylation of eNOS (Figure 5(b)).

Many studies have demonstrated deleterious effects of high-fat diets on PVAT function in experimental animals and many of these studies have made the link between oxidant stress/inflammation and NO and adiponectin formation. In a recent study published by Xia et al. [8], it was found that mice on a high-fat diet had reduced NO production by the PVAT but normal eNOS expression. Similarly, Hou et al. [14] demonstrated that high-fat diet attenuated the anticontractile effect of aortic PVAT and administration of Irisin (a myocyte-derived hormone which can cause browning of adipocytes) improved PVAT function via enhancing adiponectin and reducing superoxide and inflammatory markers within the PVAT. Calycosin, a bioactive flavonoid, even after a short ex vivo treatment has also been shown to improve PVAT function via the adiponectin/AMPK/eNOS pathway [15]. However, in most of these studies, the stress induced by the diet is of much longer duration (typically up to 20 weeks) compared to the acute effect of $\mathrm{ONOO}^{-}$addition in our study. Adiponectin may be key in understanding the effects of acute peroxynitrite which we have observed. In a recent study in atherosclerotic mice, viral delivery of adiponectin was found to reduce plaque progression and block peroxynitrite formation within the plaque [16]. It is thus possible that the response of PVAT to an acute oxidative insult such as addition of $\mathrm{ONOO}^{-}$is to upregulate adiponectin production as a defence mechanism. Future experiments may seek to clarify this by studying adiponectin gene expression following $\mathrm{ONOO}^{-}$incubation. However, to our knowledge, our study is the first to show that an oxidant agent may induce an enhancement of PVAT function in the short-term but negative effects in the longer-term. 


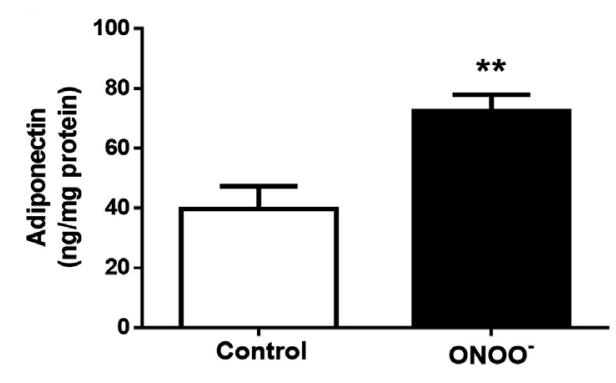

(a)

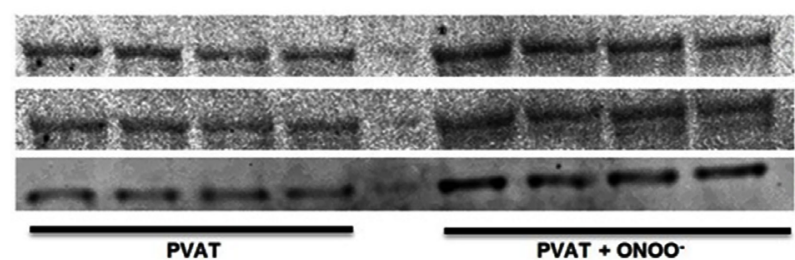

P-eNOS (Ser1177) (140kDa)

Total eNOS (140kDa)

GAPDH (37kDa)

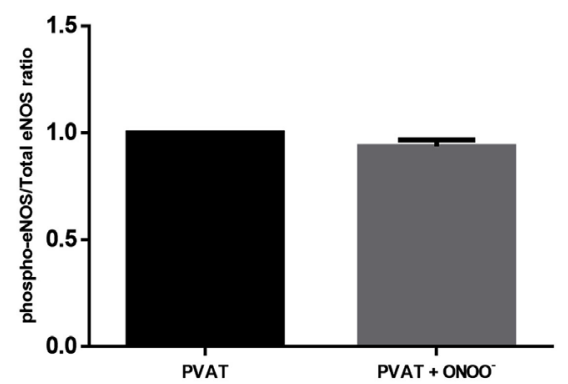

(b)

Figure 5. (a) Adiponectin content of PVAT measured by ELISA was significantly increased following treatment with $\mathrm{ONOO}^{-}\left(10^{-4} \mathrm{M}\right.$ for 60 mins; $\mathrm{n}=11$; ${ }^{* *} \mathrm{p}<0.01$ vs. control). (b) Treatment of isolated PVAT with $\mathrm{ONOO}^{-}$had no significant effect on phosphorylation of eNOS when quantified as a ratio to total eNOS; $n=4 ; p=n$.

\section{Limitation}

The main limitation of this study is that in all experiments, the PVAT has been exposed to a fairly short (1 hour) treatment with a reasonably high dose of peroxynitrite. Tissues may be exposed to such concentrations very locally at areas of active inflammation but more typically, exposure would be much longer term at lower concentrations. This would occur during disease states in conditions such as diabetes, hypertension and obesity where studies have shown that the PVAT becomes inflamed. The effect of $\mathrm{ONOO}^{-}$on PVAT under these circumstances is something which we are actively investigating.

\section{Conclusion}

In this paper we describe novel data showing the effects of an acute period of oxidant stress on the function of murine perivascular adipose tissue. We found that the oxidant radical $\mathrm{ONOO}^{-}$enhanced the ability of PVAT to exert an anticontractile effect, likely via generation of increased amounts of adiponectin by the PVAT. However, this effect was not observed if the PVAT was attached to 
the vessel, probably since the short lived $\mathrm{ONOO}^{-}$would not penetrate the vessel into the PVAT. We also found that PVAT generated both $\mathrm{NO}$ and superoxide and so $\mathrm{ONOO}^{-}$formation within the PVAT would be anticipated. Further work is required to investigate how endogenously generated $\mathrm{ONOO}^{-}$modulates PVAT function and the involvement of AMPK in these effects.

\section{Acknowledgements}

Azizah Binti Ugusman was supported by a visiting scholarship from the National University of Malaysia and Ministry of Higher. Alex Riddell was supported by an MRes/PhD studentship from the British Heart Foundation.

\section{Conflicts of Interest}

The authors declare no conflicts of interest regarding the publication of this paper.

\section{References}

[1] Almabrouk, T.A.M., Ugusman, A.B., Katwan, O.J., Salt, I.P. and Kennedy, S. (2017) Deletion of AMPK $\alpha 1$ Attenuates the Anticontractile Effect of Perivascular Adipose Tissue (PVAT) and Reduces Adiponectin Release. British Journal of Pharmacology, 174, 3398-3410. https://doi.org/10.1111/bph.13633

[2] Almabrouk, T.A.M., White, A.D., Ugusman, A.B., Skiba, D.S., Katwan, O.J., Alganga, H., Guzik, T.J., Touyz, R.M., Salt, I.P. and Kennedy, S. (2018) High Fat Diet Attenuates the Anticontractile Activity of Aortic PVAT via a Mechanism Involving AMPK and Reduced Adiponectin Secretion. Frontiers in Physiology, 9, 51. https://doi.org/10.3389/fphys.2018.00051

[3] Qi, X.Y., Qu, S.L., Xiong, W.H., Rom, O., Chang, L. and Jiang, Z.S. (2018) Perivascular Adipose Tissue (PVAT) in Atherosclerosis: A Double-Edged Sword. Cardiovascular Diabetology, 17, 134. https://doi.org/10.1186/s12933-018-0777-x

[4] Kennedy, S. and Salt, I.P. (2017) Molecular Mechanisms Regulating Perivascular Adipose Tissue-Potential Pharmacological Targets? British Journal of Pharmacology, 174, 3385-3387. https://doi.org/10.1111/bph.13969

[5] Ewart, M.A., Kennedy, S., Macmillan, D., Raja, A.L., Watt, I.M. and Currie, S. (2014) Altered Vascular Smooth Muscle Function in the ApoE Knockout Mouse during the Progression of Atherosclerosis. Atherosclerosis, 234, 154-161. https://doi.org/10.1016/j.atherosclerosis.2014.02.014

[6] Ewart, M.A., Ugusman, A., Vishwanath, A., Almabrouk, T.A.M., Alganga, H., Katwan, O.J., Hubanova, P., Currie, S. and Kennedy, S. (2017) Changes in IP3 Receptor Expression and Function in Aortic Smooth Muscle of Atherosclerotic Mice. Journal of Vascular Research, 54, 68-78. https://doi.org/10.1159/000461581

[7] Gil-Ortega, M., Stucchi, P., Guzmán-Ruiz, R., Cano, V., Arribas, S., González, M.C., Ruiz-Gayo, M., Fernández-Alfonso, M.S. and Somoza, B. (2010) Adaptative Nitric Oxide Overproduction in Perivascular Adipose Tissue during Early Diet-Induced Obesity. Endocrinology, 151, 3299-3306. https://doi.org/10.1210/en.2009-1464

[8] Xia, N., Horke, S., Habermeier, A., Closs, E.I., Reifenberg, G., Gericke, A., Mikhed, Y., Münzel, T., Daiber, A., Förstermann, U. and Li, H. (2016) Uncoupling of Endothelial Nitric Oxide Synthase in Perivascular Adipose Tissue of Diet-Induced Obese Mice. Arteriosclerosis, Thrombosis, and Vascular Biology, 36, 78-85. https://doi.org/10.1161/ATVBAHA.115.306263 
[9] da Costa, R.M., Fais, R.S., Dechandt, C.R.P., Louzada-Junior, P., Alberici, L.C., Lobato, N.S. and Tostes, R.C. (2017) Increased Mitochondrial ROS Generation Mediates the Loss of the Anti-Contractile Effects of Perivascular Adipose Tissue in High-Fat Diet Obese Mice. British Journal of Pharmacology, 174, 3527-3541. https://doi.org/10.1111/bph.13687

[10] Aghamohammadzadeh, R., Unwin, R.D., Greenstein, A.S. and Heagerty, A.M. (2015) Effects of Obesity on Perivascular Adipose Tissue Vasorelaxant Function: Nitric Oxide, Inflammation and Elevated Systemic Blood Pressure. Journal of Vascular Research, 52, 299-305. https://doi.org/10.1159/000443885

[11] Marchesi, C., Ebrahimian, T., Angulo, O., Paradis, P. and Schiffrin, E.L. (2009) Endothelialnitric Oxide Synthase Uncoupling and Perivascular Adipose Oxidative Stress and Inflammation Contribute to Vascular Dysfunction in a Rodent Model of Metabolic Syndrome. Hypertension, 54, 1384-1392. https://doi.org/10.1161/HYPERTENSIONAHA.109.138305

[12] Costa, R.M., Filgueira, F.P., Tostes, R.C., Carvalho, M.H., Akamine, E.H. and Lobato, N.S. (2016) $\mathrm{H}_{2} \mathrm{O}_{2}$ Generated from Mitochondrial Electron Transport Chain in Thoracic Perivascular Adipose Tissue Is Crucial for Modulation of Vascular Smooth Muscle Contraction. Vascular Pharmacology, 84, 28-37. https://doi.org/10.1016/j.vph.2016.05.008

[13] Bussey, C.E., Withers, S.B., Saxton, S.N., Bodagh, N., Aldous, R.G. and Heagerty, A.M. (2018) $\beta$ (3)-Adrenoceptor Stimulation of Perivascular Adipocytes Leads to Increased Fat Cell-Derived NO and Vascular Relaxation in Small Arteries. British Journal of Pharmacology, 175, 3685-3698. https://doi.org/10.1111/bph.14433

[14] Hou, N., Liu, Y., Han, F., Wang, D., Hou, X., Hou, S. and Sun, X. (2016) Irisin Improves Perivascularadipose Tissue Dysfunction via Regulation of the Heme Oxygenase-1/Adiponectinaxis in Diet-Induced Obese Mice. Journal of Molecular and Cellular Cardiology, 99, 188-196. https://doi.org/10.1016/j.yjmcc.2016.09.005

[15] Han, F., Li, K., Pan, R., Xu, W., Han, X., Hou, N. and Sun, X. (2018) Calycosin Directly Improves Perivascular Adipose Tissue Dysfunction by Upregulating the Adiponectin/AMPK/Enospathway in Obese Mice. Food \& Function, 9, 2409-2415. https://doi.org/10.1039/C8FO00328A

[16] Cai, X., Li, X., Li, L., Huang, X.Z., Liu, Y.S., Chen, L., Zhang, K., Wang, L., Li, X., Song, J., Li, S., Zhang, Y. and Zhang, M. (2015) Adiponectin Reduces Carotid Atherosclerotic Plaque Formationin ApoE-/-Mice: Roles of Oxidative and Nitrosative Stress and Inducible Nitricoxide Synthase. Molecular Medicine Reports, 11, 1715-1721. https://doi.org/10.3892/mmr.2014.2947 